\title{
Vaccination With ENO1 DNA Prolongs Survival of Genetically Engineered Mice With Pancreatic Cancer
}

\author{
PAOLA CAPPELLO, ${ }^{1,2}$ SIMONA ROLLA, ${ }^{1,2}$ ROBERTO CHIARLE, ${ }^{1}$ MOITZA PRINCIPE, ${ }^{1,2}$ FEDERICA CAVALLO, ${ }^{2,3}$ \\ GIOVANNI PERCONTI, ${ }^{4,5}$ SALVATORE FEO, ${ }^{4,5}$ MIRELLA GIOVARELLI, ${ }^{1,2}$ and FRANCESCO NOVELLI ${ }^{1,2}$
}

${ }^{1}$ Center for Experimental Research and Medical Studies, Città della Salute e della Scienza di Torino, ${ }^{2}$ Department of Molecular Biotechnology and Health Science, and ${ }^{3}$ Molecular Biotechnology Center, University of Turin, Turin; ${ }^{4}$ Department of Experimental Oncology and Clinical Applications, University of Palermo, Palermo; and ${ }^{5}$ Institute of Biomedicine and Molecular Immunology, National Council of Research, Palermo, Italy

\section{See Covering the Cover synopsis on page 862.}

BACKGROUND \& AIMS: Pancreatic ductal adenocarcinoma (PDA) is an aggressive tumor, and patients typically present with late-stage disease; rates of 5-year survival after pancreaticoduodenectomy are low. Antibodies against $\alpha$-enolase (ENO1), a glycolytic enzyme, are detected in more than $60 \%$ of patients with PDA, and ENO1-specific $T$ cells inhibit the growth of human pancreatic xenograft tumors in mice. We investigated whether an ENO1 DNA vaccine elicits antitumor immune responses and prolongs survival of mice that spontaneously develop autochthonous, lethal pancreatic carcinomas. METHODS: We injected and electroporated a plasmid encoding ENO1 (or a control plasmid) into $\mathrm{Kras}^{\mathrm{G} 12 \mathrm{D}} / \mathrm{Cre}$ (KC) mice and $\mathrm{Kras}^{\mathrm{G} 12 \mathrm{D}} / \mathrm{Trp} 53^{\mathrm{R} 172 \mathrm{H}} / \mathrm{Cre}(\mathrm{KPC})$ mice at 4 weeks of age (when pancreatic intraepithelial lesions are histologically evident). Antitumor humoral and cellular responses were analyzed by histology, immunohistochemistry, enzyme-linked immunosorbent assays, flow cytometry, and enzyme-linked immunosorbent spot and cytotoxicity assays. Survival was analyzed by Kaplan-Meier analysis. RESULTS: The ENO1 vaccine induced antibody and a cellular response and increased survival times by a median of 138 days in KC mice and 42 days in KPC mice compared with mice given the control vector. On histologic analysis, the vaccine appeared to slow tumor progression. The vaccinated mice had increased serum levels of anti-ENO1 immunoglobulin G, which bound the surface of carcinoma cells and induced complement-dependent cytotoxicity. ENO1 vaccination reduced numbers of myeloid-derived suppressor cells and T-regulatory cells and increased T-helper 1 and 17 responses. CONCLUSIONS: In a genetic model of pancreatic carcinoma, vaccination with ENO1 DNA elicits humoral and cellular immune responses against tumors, delays tumor progression, and significantly extends survival. This vaccination strategy might be developed as a neoadjuvant therapy for patients with PDA.

Keywords: Th17; IFN- $\gamma$; Antitumor Immunity; Immunotherapy.
Dancreatic ductal adenocarcinoma (PDA) is the fourth leading cause of cancer-related death in Western countries. Surgical resection is the only potentially curative treatment. Unfortunately, because of the late presentation of the disease, only $15 \%$ to $20 \%$ of patients are candidates for pancreatectomy. However, the 5-year survival rate after pancreaticoduodenectomy is only $25 \%$ to $30 \%$ for node-negative and $10 \%$ for node-positive tumors. ${ }^{1,2}$ Effective diagnostic and therapeutic strategies are still urgently needed to improve this survival rate. We have used SERological Proteome Analysis to identify a dozen antigens expressed by PDA and recognized by autoantibodies present in the sera of patients with pancreatic cancer but not in the sera of patients with other tumors, patients with pancreatitis, or healthy donors. ${ }^{3}$ One of these antigens, $\alpha$-enolase (ENO1), is specifically recognized by more than $60 \%$ of patients with PDA. ${ }^{4}$ ENO 1 is coded by the ENO1 gene, is overexpressed in the cytoplasm of PDA cells, and is also present on their membrane. ${ }^{5}$ In the cytoplasm, ENO1 acts as a glycolytic enzyme, whereas on the membrane it acts as a plasminogen receptor and plays an important role in cell migration. 6,7 We have shown that patients with PDA with autoantibodies to ENO1 also present an ENO1-specific T-cell response that is not observed in patients with no ENO1 autoantibodies. On transfer into immunocompromised mice, ENO1-specific $\mathrm{T}$ cells inhibit the growth of xenotransplanted human pancreatic tumors. Despite the ubiquitous presence of ENO1 in all mammalian cells, normal cells expressing low levels of ENO1 are spared by ENO1specific cytotoxic $\mathrm{T}$ lymphocytes. ${ }^{5}$

In this work, we used 2 genetically engineered mice strains (Kras ${ }^{\mathrm{G} 12 \mathrm{D}} / \mathrm{Cre}$ mice [KC mice] and $\mathrm{Kras}^{\mathrm{G} 12 \mathrm{D}}$ / $\operatorname{Trp} 53^{\mathrm{R} 172 \mathrm{H}} / \mathrm{Cre}$ mice [KPC mice]) that develop autochthonous lethal pancreatic carcinomas with different kinet-

Abbreviations used in this paper: BSA, bovine serum albumin; ENO1, $\alpha$-enolase; IFN, interferon; Ig, immunoglobulin; IL, interleukin; KC, $\mathrm{Kras}^{\mathrm{G} 12 \mathrm{D}}$ /Cre mice; KPC, Kras ${ }^{\mathrm{G} 12 \mathrm{D}} / \mathrm{Trp} 53^{\mathrm{R} 172 \mathrm{H}} /$ Cre mice; mAb, monoclonal antibody; MDSC, myeloid-derived suppressor cells; PBS, phosphate-buffered saline; PDA, pancreatic ductal adenocarcinoma; rENO1, recombinant human ENO1; TNF, tumor necrosis factor; Treg cell, T-regulatory cell.

\section{(1) 2013 by the AGA Institute $0016-5085 / \$ 36.00$}

http://dx.doi.org/10.1053/j.gastro.2013.01.020 
$\mathrm{ics}^{8,9}$ to study the protective effect of a DNA vaccine to human ENO1.

Mice were vaccinated with plasmids encoding human ENO1 because it displays more than 95\% identity (99\% homology) with the mouse orthologue. Vaccination, starting from 4 weeks of age, when pancreatic intraepithelial lesions are already histologically evident, ${ }^{8}$ elicits an integrated humoral and cellular immune response to ENO1 that significantly extends survival. Our data also show a new role of ENO1 in skewing the T-cell response toward a Th17-type response. ENO1 vaccination may therefore be a promising neoadjuvant form of PDA management.

\section{Materials and Methods}

\section{Mice}

Mice carrying single-mutated $\mathrm{Kras}^{\mathrm{G} 12 \mathrm{D}}$ (C57BL/6;129SvJae $\mathrm{H}-2^{\mathrm{b}}$ ) or double-mutated (Kras ${ }^{\mathrm{G} 12 \mathrm{D}}$ and $\left.\operatorname{Trp} 53^{\mathrm{R} 172 \mathrm{H}}\right)\left(129 \mathrm{SvJae} \mathrm{H}-2^{\mathrm{b}}\right)$ under the endogenous promoter and flanked by Lox-STOP-Lox cassettes were obtained from Dr David Tuveson (Cancer Research UK, Cambridge Research Institute, Cambridge, England). C57BL/6 mice expressing Cre recombinase under a specific pancreatic transcriptional factor, pancreatic duodenum homeobox 1 (Pdx-1), promoter were obtained from Dr Andrew Lowy (University of San Diego, San Diego, CA). Mice were bred and maintained under saprophytic and pathogen-free conditions at the animal facilities of the Molecular Biotechnology Center and treated in accordance with EU and institutional guidelines. Pancreatic cancer-prone KC and KPC mice were generated by crossing single-mutated $\mathrm{Kras}^{\mathrm{G} 12 \mathrm{D}}$ or double-mutated $\mathrm{Kras}^{\mathrm{G} 12 \mathrm{D}}$ and $\operatorname{Trp} 53^{\mathrm{R} 172 \mathrm{H}}$ with $\mathrm{C} 57 \mathrm{BL} / 6$ mice expressing Cre recombinase. Mice were screened by polymerase chain reaction using tail DNA amplified by specific primers to the Lox-P cassette flanking Kras and wild-type Kras genes, the Lox-P cassette flanking Trp53 mutated and wild-type Trp53 and Cre recombinase genes. Polymerase chain reaction products were separated on $1.5 \%$ agarose gels with GelRed (Biotum by SIC, Rome, Italy) and recorded as .tiff.

\section{DNA Vaccination}

$\mathrm{KC}$ and KPC mice were vaccinated at 4 weeks of age and every 3 weeks for a total of 3 rounds of vaccination or every 2 weeks for a total of 4 rounds of vaccination. In the therapeutic DNA vaccination setting, KC mice were vaccinated at 32 to 36 weeks of age and every 3 weeks for a total of 3 rounds of vaccination. Injection of $50 \mu \mathrm{g}$ of plasmid in $40 \mu \mathrm{L}$ of sterile water with $0.9 \% \mathrm{NaCl}$ into the femoral muscle of mice anesthetized with Zoletil (Rompun) e Xylazina was immediately followed by two 25 -millisecond pulses of $375 \mathrm{~V} / \mathrm{cm}$ applied with a Cliniporator and linear needle electrodes (IGEA, Carpi, Italy). KC and KPC mice of the same age were randomly assigned to control and treatment groups, and all groups were specifically treated concurrently. Mice were monitored weekly and left to live unless showing obvious signs of pain to obtain a Kaplan-Meier survival curve. Parallel mice were killed at 4, 24, and 36 weeks of age as indicated to perform histologic or immunohistochemical analyses.

Human ENO1 complementary DNA was obtained by enzyme digestion of the plasmid pRC-ENO110 (kindly provided by A. Giallongo, Institute of Biomedicine and Molecular Immunology, National Council of Research, Palermo, Italy) in HindIII and XbaI restriction sites (both from M-Medical, Milan, Italy), followed by separation by electrophoresis on agarose gel and elu- tion. It was then cloned into pVAX1 (Invitrogen, Milan, Italy), previously digested with the same restriction enzymes, by ligation. To propagate and maintain empty and PVAX-ENO1 vectors, the competent recA1, endA Escherichia coli strain (TOP10) was transformed with the empty PVAX and ligation mixture and selected on Luria Bertoni plates containing $50 \mu \mathrm{g} / \mathrm{mL}$ kanamycin.

\section{Cells}

Syngeneic murine DT6606 and K8484 cells were kindly provided by Dr K. Olive (Li Ka Shing Centre, Cambridge Research Institute, Cancer Research UK, Cambridge, England). They were obtained from a $\mathrm{Kras}^{\mathrm{G} 12 \mathrm{D}} / \mathrm{Cre}$ and a $\mathrm{Kras}^{\mathrm{G} 12 \mathrm{D}} /$ $\operatorname{Trp} 53^{\mathrm{R} 172 \mathrm{H}} / \mathrm{Cre}$ pancreatic tumor mass, respectively, and were maintained in vitro in Dulbecco's modified Eagle medium/10\% fetal bovine serum.

\section{Enzyme-Linked Immunosorbent Assay}

Anti-ENO1 immunoglobulin (Ig) G was measured by enzyme-linked immunosorbent assay by binding to recombinant human ENO1 (rENO1) $\left(1.5 \mu \mathrm{g} / \mathrm{mL}\right.$ in $\mathrm{Na}_{2} \mathrm{CO}_{3} 0.1 \mathrm{~mol} / \mathrm{L}$ ), produced as previously described. ${ }^{5}$ Sera collected 2 weeks after 3 rounds of vaccination were diluted 1:500 in phosphate-buffered saline (PBS) and antibody concentration was calculated by regression analysis using 8 two-fold serial dilutions of $1 \mu \mathrm{g} / \mathrm{mL}$ of $72 / 1.11$ monoclonal antibody $(\mathrm{mAb})$ for a standard curve (kindly provided by P. Migliorini, University of Pisa, Pisa, Italy).

Serum-binding potential. Sera from untreated and empty or ENO1-vaccinated mice were used to stain DT6606 or K8484 cells, which were analyzed by flow cytometry after dilution of 1:50. Briefly, $1 \times 10^{5}$ cells were washed with $\mathrm{PBS} / 0.2 \%$ bovine serum albumin (BSA) $/ 0.01 \% \mathrm{NaN}_{3}$ and incubated with diluted sera for 1 hour at $4^{\circ} \mathrm{C}$. After 2 washes, cells were incubated with an APC-conjugated anti-mouse antibody (1:200; Biolegend; Prodotti Gianni, Milan, Italy) for 30 minutes on ice. Following washing, 10,000 cells were acquired with a FACSCanto using CellQuest software (both BD Biosciences, Buccinasco, MI, Italy). The antibody titer is expressed as serum binding potential $\times 10^{-3} / \mathrm{mL}$, calculated as previously described in detail. ${ }^{11}$

Complement-dependent cytotoxicity. DT6606 or K8484 cells were seeded in a 96-well plate $\left(5 \times 10^{3} /\right.$ well $)$ in Dulbecco's modified Eagle medium/1\% fetal bovine serum overnight for adhesion. Cells were washed with warm PBS, incubated with sera diluted in PBS (1:50) for 1 hour at $4^{\circ} \mathrm{C}$, and washed again, followed by incubation with fresh reconstituted rabbit complement (Low-Tox rabbit complement; Cedarlane; Euroclone, Milan, Italy) diluted 1:25 in PBS for 1 hour at $37^{\circ} \mathrm{C}$. Lysis was evaluated with the CytoTox 96 Non-Radioactive Cytotoxicity Assay (Promega, Milan, Italy). Lysis buffer was added to cells 45 minutes before centrifugation to obtain the maximum release of lactate dehydrogenase, while cells without serum and complement were used as a measure of spontaneous release of lactate dehydrogenase. Plates were centrifuged at $250 g$ for 4 minutes and $50 \mu \mathrm{L}$ of supernatant was transferred to the enzymatic assay plate and incubated with $50 \mu \mathrm{L}$ of substrate mix for 30 minutes at room temperature in the dark. Stop solution $(50 \mu \mathrm{L})$ was added to each well, and absorbance was recorded at $490 \mathrm{~nm}$ with a plate reader. A lactate dehydrogenase positive control was added in new wells of each plate, and all tests were performed in triplicate. The percentage of specific lysis was calculated using the following formula: \% Cytotoxicity = ([Experimental - Target Spontaneous]/[Target Maximum Target Spontaneous]) $\times 100$. 


\section{Interferon Gamma Enzyme-Linked Immunosorbent Spot Assay}

Mouse lymph node and spleen cells were evaluated to determine the presence of $\mathrm{T}$ cells able to secrete interferon (IFN)- $\gamma$ in response to rENO1 or DT6606 cells ex vivo or after 1 week of in vitro culture in the presence of $10 \mu \mathrm{g} / \mathrm{mL}$ of rENO1. Nitrocellulose plates (Millipore, Milan, Italy) were coated with anti-IFN- $\gamma$ capture mAb (mIFN- $\gamma$ kit; BD Biosciences) overnight at $4{ }^{\circ} \mathrm{C}$. $\mathrm{T}$ cells from lymph nodes and spleens ex vivo or recovered from a 1-week culture were stimulated with DT6606 cells $(1: 10=\mathrm{S} / \mathrm{E})$ or $\mathrm{rENO} 1$ for 40 hours at $37^{\circ} \mathrm{C}$. T cells were seeded at $3 \times 10^{5}$ cells/well, and all conditions were performed in quadruplicate. Plates were then developed as indicated by the manufacturer using AEC (Sigma-Aldrich, Milan, Italy) substrate, and spots were quantified with the microplated reader along with a computer-assisted image analysis system (AID; Amplifon, Milan, Italy). The number of spots was calculated by subtracting the number of spots in medium only (background) from that in the presence of stimuli.

\section{Flow Cytometry}

Mouse myeloid-derived suppressor cells (MDSCs) were analyzed by staining whole blood after red cell lysis with $0.83 \%$ $\mathrm{NH}_{4} \mathrm{Cl} / 0.1 \% \mathrm{KHCO}_{3} / 0.04 \%$ EDTA buffer and washing with PBS/ $0.2 \% \mathrm{BSA} / 0.01 \% \mathrm{NaN}_{3}$. This was followed, after blocking nonspecific sites, by incubation with the following mAbs from Biolegend: anti-CD16/CD32 mAb, anti-CD11b, and anti-Gr1. Mouse peripheral blood mononuclear cells, isolated by Ficoll centrifugation, were washed with $\mathrm{PBS} / 0.2 \% \mathrm{BSA} / 0.01 \% \mathrm{NaN}_{3}$, stained with CD4 and CD25 mAbs (all from Biolegend), and subsequently fixed and permeabilized with Fixation and Permeabilization Solution (eBioscience; Campoverde, Milan, Italy) for 30 minutes at $4^{\circ} \mathrm{C}$. After washing with permeabilization buffer, cells were incubated for 30 minutes with FoxP3 and RoR $\gamma \mathrm{t}$ $\mathrm{mAbs}$ (both from eBioscience). Spleen cells $\left(2 \times 10^{6} / \mathrm{mL}\right)$ from control or ENO1-vaccinated mice were stimulated with phorbol myristate acetate $(50 \mathrm{ng} / \mathrm{mL})$ and ionomycin $(2 \mu \mathrm{g} / \mathrm{mL})$ in the presence of the intracellular protein transport inhibitor brefeldin A (Sigma-Aldrich) ex vivo or after 1 week of culture in the presence of rENO1. Cells were harvested 5 hours later, labeled with a CD4 $\mathrm{mAb}$, and subsequently fixed with a $2 \%$ paraformaldehyde solution. Cells were permeabilized using PBS/0.2\% BSA/ $0.5 \%$ saponin and subsequently incubated for 30 minutes with mAbs specific for tumor necrosis factor (TNF) $-\alpha$, IFN- $\gamma$, and interleukin (IL)-17 (all from BD or Biolegend). All flow cytometry data were acquired on a FACSCalibur (BD Biosciences) and analyzed using FlowJo (Tree Star from BD Biosciences) or CellQuest software (BD Biosciences).

\section{Histology}

Pancreas, spleen, liver, and lungs from control and ENO1-vaccinated mice were sampled at the indicated times, fixed in formalin, and subsequently paraffin embedded. We quantified the percentage of transformed ducts compared with normal ducts on histologic sections from H\&E-stained sections of the pancreas, according to the criteria previously established. ${ }^{8}$

For immunohistochemical analysis, slides were subjected to microwaving for 20 minutes in $10 \mathrm{mmol} / \mathrm{L}$ of citrate buffer $(\mathrm{pH}$ 8.0 for nuclear antigens; $\mathrm{pH} 6.0$ for other antigens). Immunostaining was performed using the avidin biotin peroxidase complex method or detected using the Dako Envision Plus Rabbit Polymer (K4033) and a semiautomated immunostainer (Dako, Carpinteria, CA, or Ventana Systems, Tucson, AZ). The primary antibody used was a rat anti-mouse FoxP3 1:50 (eBioscience) and a rat anti-mouse CD3 1:100 (Dako). Reactive T lymphocytes and T-regulatory (Treg) cells were quantified by measuring the percentages of $\mathrm{CD}^{+}$and $\mathrm{FoxP}^{+}$cells, respectively, among the total mononuclear cells infiltrating the neoplastic pancreatic glands.

\section{Statistical Analysis}

We used an unpaired 2-tailed Student $t$ test for all comparisons. Kaplan-Meier survival curves were created with GraphPad software (Prism 5, La Jolla, CA) and evaluated with both the log-rank Mantel-Cox and the Gehan-Breslow-Wilcoxon test.

\section{Results}

\section{ENO1 Vaccine Induces Both an Antibody and a Cellular Response}

PDA-prone KC mice were electroporated either with empty plasmid or human ENO1-encoding plasmid. The amount of antibodies able to bind rENO1 was evaluated at 2 weeks after the last electroporation. Anti-ENO1 antibodies were significantly induced in ENO1-vaccinated $\mathrm{KC}$ mice, but not in those vaccinated with the empty vector (Figure $1 A$ ).

To evaluate the functional role of anti-ENO1 antibodies, we first analyzed the ability of sera from empty- or ENO1-vaccinated mice to bind the cell surface of murine PDA cells by flow cytometry by measuring their binding potential (Figure $1 B-D$ ). Despite a weak cell decoration also being observed with sera from untreated mice, those from ENO1-vaccinated mice displayed higher serum binding potential (Figure 1B,D) and a significantly higher ability to mediate complement-dependent killing of both murine PDA K8484 and DT6606 cells (Figure 1D, E).

Spleen and lymph node cells from untreated, emptyvaccinated, and ENO1-vaccinated mice were collected 2 weeks after the final vaccination, and their ability to secrete IFN- $\gamma$ was assessed in an enzyme-linked immunosorbent spot assay, both ex vivo and after 7 days of in vitro restimulation with the rENO1. Ex vivo splenocytes from untreated and empty-vaccinated control mice (white and gray bars) displayed few specific spots when stimulated with rENO1 (Figure $2 B$ and $C$ ). In contrast, ex vivo $T$ cells from ENO1-vaccinated mice (black bars) displayed a significantly higher number of IFN- $\gamma$-secreting cells in response to rENO1 (Figure 2B), which increased 3-fold after the in vitro rENO1 restimulation (Figure 2D). When DT6606 cells were used for stimulation, only rENO1restimulated $\mathrm{T}$ cells from ENO1-vaccinated mice specifically secreted IFN- $\gamma($ Figure 1E). No IFN- $\gamma$-secreting cells appeared when DT6606 cells were preincubated with an anti-major histocompatibility complex class I antibody. Similar results were obtained with lymph node cells (data not shown).

\section{ENO1 DNA Vaccine Prolongs Mouse Survival}

Because electroporation of human ENO1-encoding plasmid induces both cellular and antibody-mediated 


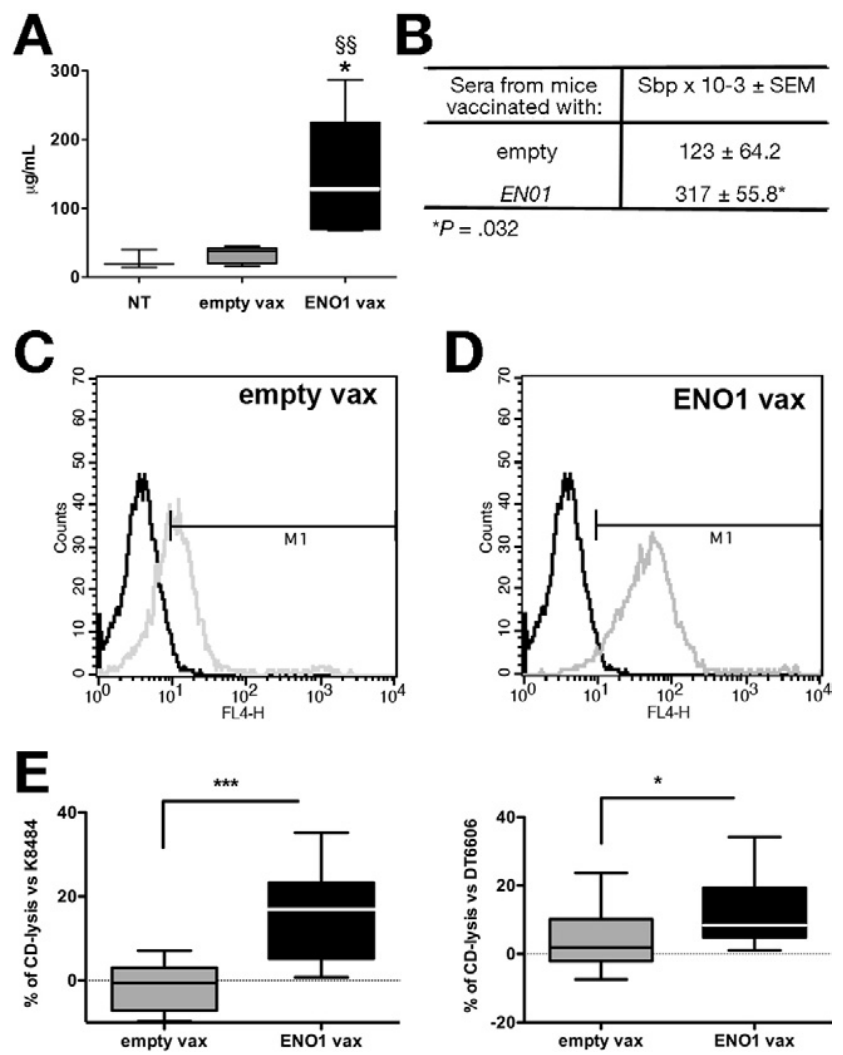

Figure 1. Electroporation of human ENO1-encoding plasmid elicited antibodies to human and mouse ENO1. (A) Anti-ENO1 IgG was quantified by enzyme-linked immunosorbent assay in sera from untreated (white bars) and empty- (gray bars) or ENO1-plasmid (black bars) vaccinated mice after 3 rounds of vaccination. Data are represented as mean \pm SEM. (B) Serum binding potential evaluated by flow cytometry with empty- or ENO1-vaccinated mouse sera collected 2 weeks after the final vaccination. Values are expressed as mean $\times 10^{-3} \pm$ SEM from 8-10 mice. *Values from ENO1-vaccinated mice are significantly different from those of empty-vaccinated mice. Representative staining of murine DT6606 PDA cells with sera from $(C)$ empty- or (D) ENO1-vaccinated mice that were individually tested. Open profiles, cells stained with secondary antibody alone; solid black profiles, cells stained with sera from mice vaccinated with empty (left pane/) or ENO1 (right panel) plasmids. Two representative stainings are shown. (E) Complement-dependent cytotoxicity of K8484 and DT6606 cells with sera from empty- or ENO1-vaccinated mice that were individually tested. The box graphs include single percent of lysis \pm SEM, and the horizontal bars represent the median for each group of sera. ${ }^{\star} P<.05$ and ${ }^{* \star *} P<.0001$ values from ENO1-vaccinated mice are significantly different from those of empty-vaccinated mice. $\S \S P<.001$.

immune reactions, the therapeutic efficacy of this response was evaluated. As shown in Figure $3 A$ and $B$, almost all $\mathrm{KC}$ mice displayed transformed foci in the pancreas at the moment of the first electroporation. Their number increased until the tumor mass reached $85 \%$ to $100 \%$ of the pancreas, and $50 \%$ of mice died around 336 days of age due to the presence of large tumors (Figure $3 C)$. The vaccination with empty vector slightly prolonged the median of survival by 56 days $(P=.9$; log-rank Mantel-Cox test). In ENO1-vaccinated mice, the median survival was extended by 140 days $(50 \%$ of mice died around 474 days; $P=.033$ vs untreated mice; log-rank Mantel-Cox test) (Figure 3C), which amounts to more than one-third of their life expectancy. Despite the slight increase induced by the unspecific vaccination and electroporation, the ENO1 vaccine significantly prolonged the survival by a further 82 days $(P=.036$ vs empty-vaccinated mice; log-rank Mantel-Cox test).

Even the survival of the double-mutated KPC mice, in which faster tumor progression was evident from their shorter median survival (203 days compared with 336 days for KC mice), was prolonged by 42 days ( $20 \%$ of life expectancy; $P=.034$ vs untreated mice and $P=.025$ vs empty-vaccinated mice; log-rank Mantel-Cox test) in ENO1-vaccinated mice but not in empty-vaccinated mice (50\% of mice died around 160 days; $P=.25$ vs untreated mice; log-rank Mantel-Cox test) (Figure 3D).

Because of the small number of available KPC mice, histologic and immunologic studies were conducted on $\mathrm{KC}$ mice only. Histologic analysis performed at 24 and 36 weeks of age with randomly vaccinated mice $(\mathrm{n}=5$ per group) confirmed the ability of the ENO1 vaccine, and to a lesser extent electroporation with the empty plasmid, to reduce the percentage of transformed ducts compared with control mice. At 24 weeks, while untreated $\mathrm{KC}$ mice displayed $58 \%$ of already transformed pancreatic ducts, those empty vaccinated or those receiving ENO1 vaccine displayed only $18 \%$ and $25.5 \%$, respectively (Figure $3 E$ ). At 36 weeks, ducts were shown to be transformed in $85 \%$ of the pancreas of untreated mice and 56\% in those empty vaccinated compared with only $40 \%$ in those ENO1 vaccinated (Figure 3F); 2 of these mice displayed an almost normal pancreas, suggesting a complete recovery.

\section{ENO1 DNA Vaccine Inbibits MDSC and Treg Cell Expansion and Promotes the Th17 Response}

Because MDSCs and Treg cells have been reported in $\mathrm{KC}$ mice, ${ }^{12}$ the effect of ENO1 DNA vaccination on these cell subsets was investigated. Compared with control mice at 12 weeks of age, there was a similar percentage of MDSCs (white bars, Figure 4A, left panel) in the blood of empty-vaccinated mice (gray bars), which significantly decreased in ENO1-vaccinated mice (black bars), with an even more pronounced effect in Treg cells (Figure $4 A$, right panel). The percentages of MDSCs and Treg cells in ENO1-vaccinated mice then increased to those of control mice at 52 and 76 weeks (Figure $4 A$ ).

Immunohistochemical examination of pancreatic tissues from untreated mice shows a progressive increase of $\mathrm{FoxP}^{+}$cells in transformed ducts from $3 \%$ at 4 weeks of age to $13 \%$ at 24 weeks and $19 \%$ at 36 weeks. Empty vaccination did not alter this progress, whereas the ENO1 vaccine significantly diminished both the 24 - and 36-week percentages (Figure $4 B-F$ ). It will be seen that these 2 percentages are stable.

Compared with untreated and empty-vaccinated mice, in ENO1-vaccinated mice the percentage of Treg cells decreased in parallel with the increase of the percentage of cells expressing RoR $\gamma t$, a transcriptional factor related to Th17 cells ${ }^{13}$ (data not shown). This corresponded to an 
A
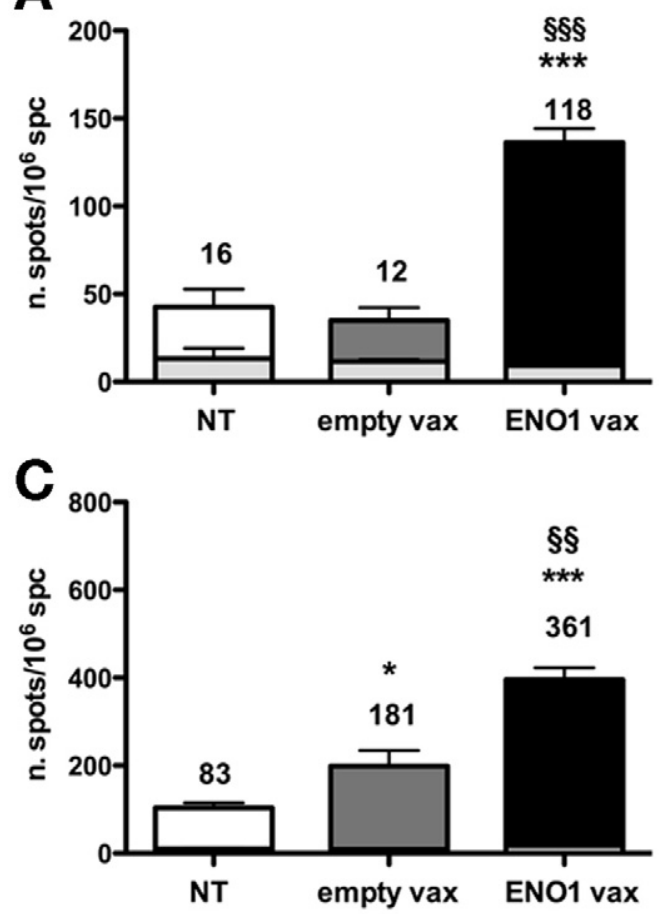

$\mathbf{B}$

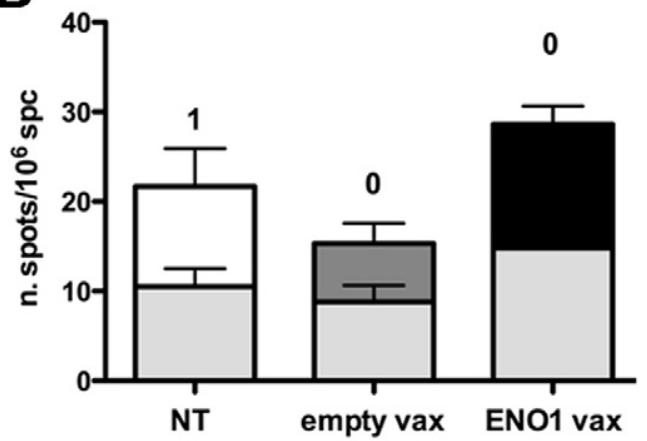

D

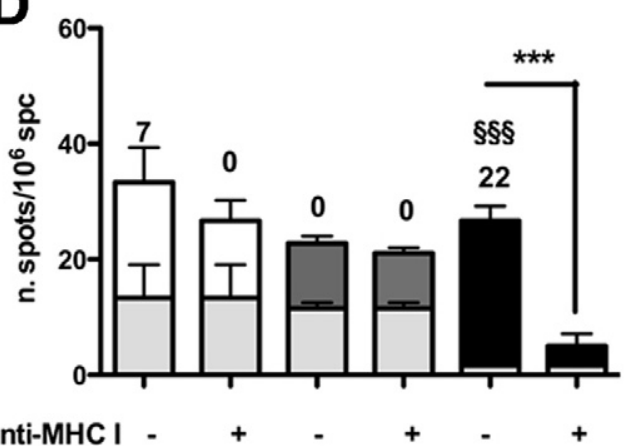

Figure 2. ENO1 vaccine elicited cellular responses to human and mouse ENO1. Spleen cells from untreated mice (white bars), empty plasmid (gray bars), or ENO1 plasmid-vaccinated (black bars) mice were stimulated on an enzyme-linked immunosorbent spot plate with ( $A$ and $C)$ rENO1 or ( $B$ and $D)$ DT6606 cells $(A$ and $B)$ ex vivo or $(C$ and $D)$ after 7 days of stimulation with rENO1. DT6606 cells were either preincubated or not with anti-major histocompatibility complex class I mAb $(D)$. T cells cultured with medium alone are shown as light gray bars. The numbers in the graph represent the mean number of specific spots subtracted from that of the background. All conditions were in quadruplicate. One of 3 independent experiments is shown, and results are expressed as mean \pm SEM. $\mathrm{n}=2$ for each group. ${ }^{\star} P<.05,{ }^{\star \star *} P<.0001$ values compared with untreated mice and $\$ \S P<.001, \S \S \$ P<.0001$ values compared with empty-vaccinated mice.

increased percentage of cells secreting IL-17 and TNF- $\alpha, 2$ signature cytokines of Th17 cells, ${ }^{14}$ accompanied by an increase of IFN- $\gamma$-secreting cells in $\mathrm{CD}^{+}$spleen cells from $E N O 1$-vaccinated mice (Figure $5 A$ ). After 7 days of in vitro restimulation, the percentage of IL-17-, TNF- $\alpha-$, IFN- $\gamma$-, and IL-17/TNF- $\alpha$-secreting cells significantly increased even further (Figure 5B). Furthermore, we analyzed by immunohistochemistry the CD3 infiltrate into neoplastic foci from pancreas collected from untreated mice and mice vaccinated with empty or ENO1-expressing plasmids. As shown in Figure 5C-F, the percentage of $\mathrm{CD} 3$ on total inflammatory cells in the neoplastic foci was significantly higher in ENO1-vaccinated mice compared with that observed in empty-vaccinated or untreated mice.

These results suggest that only the ENO1 vaccination was able to induce specific Th17 cells in parallel, to diminish the frequency of suppressor cells such as MDSCs and Treg cells, and, of note, to actively recruit CD3 cells into the tumor.

\section{Therapeutic ENO1 DNA Vaccine Significantly Slows Progression of PDA}

To evaluate the effect of the ENO1 vaccine in a setting closer to that applicable in patients with late diagnosed or chemoresistant or radioresistant tumors, we vaccinated mice at 32 to 36 weeks of age. Mice were killed at 52 weeks of age to evaluate by histologic analysis the percentage of transformed ducts compared with the normal ducts. Empty-vaccinated mice showed approximately $79 \%$ of transformed ducts compared with approximately $50 \%$ observed in the pancreas of ENO1-vaccinated mice (Figure 6A). Although the difference in the percent of transformed ducts was not statistically different, the mean dimension of the largest tumor is strongly and significantly less in ENO1-vaccinated mice compared with that evaluated in empty-vaccinated mice (Figure 6B). These results suggest that even in a desperate attempt to tackle PDA when tumors are well established, the ENO1 vaccine seems to have efficacy in delaying tumor progression.

\section{Discussion}

We have previously shown that ENO1, a novel PDA-associated antigen, could be a promising therapeutic candidate owing to its ability to induce an integrated humoral and cellular response. ${ }^{5}$ The few PDA-associated antigens (CEA, Kras, MUC1, and gastrin) that have already been tested in clinical trials have been shown to have no effect on survival. ${ }^{15}$ This highlights the challenge to identify new and more significant immunogenic targets.

Here we show, for the first time, that a DNA vaccine coding for a ubiquitous protein significantly induces a 

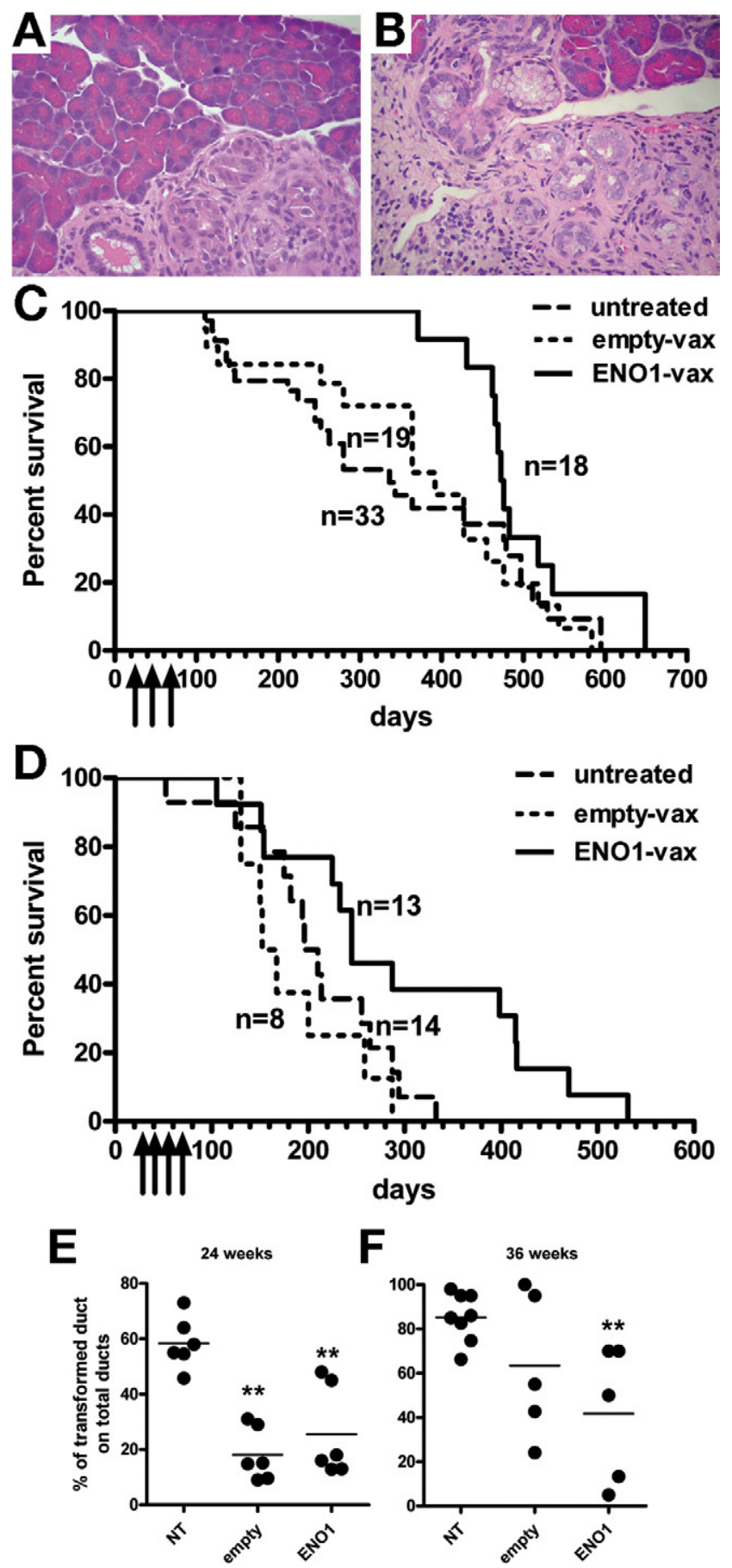

Figure 3. ENO1 vaccine significantly prolonged survival of $\mathrm{KC}$ and $\mathrm{KPC}$ mice. ( $A$ and $B$ ) From 4 weeks of age, $\mathrm{KC}$ mice spontaneously developed PDA that histologically progressed from PanIN to carcinoma in situ. (C and $D$ ) Kaplan-Meier analysis of survival of untreated and vaccinated with empty or human ENO1 coding plasmid (C) KC mice and (D) KPC mice monitored until their death. Percentage of transformed ducts in $\mathrm{KC}$ mice untreated or vaccinated with empty or ENO1 DNA at $24(E)$ and 36 $(F)$ weeks. ${ }^{* \star} P<.001$ values compared with untreated mice.

specific immune response that prolongs survival in a mouse model of PDA. Despite ENO1 being widely expressed, we have previously shown that normal cells, whose ENO1 levels are lower than those of tumor cells, are spared from antigen-specific killing. ${ }^{5}$
In this study, LSL-Kras ${ }^{\mathrm{G} 12 \mathrm{D}}$ mice crossed with Pdx-1-Cre mice (KC mice) were used to obtain the specific expression of mutated $\mathrm{Kras}^{\mathrm{G} 12 \mathrm{D}}$ in pancreatic cells. Each tumor evolves from a background of genomic instability that gives rise to a polyclonal tumor with physiopathological features that are similar to those of human PDA. ${ }^{8}$ Indeed, high-resolution assessments of chromosomal content have previously indicated that nonreciprocal translocations were found in most neoplastic cells that were analyzed. ${ }^{16}$ This and similar models of genetically engineered mice have been used to address therapeutic issues but

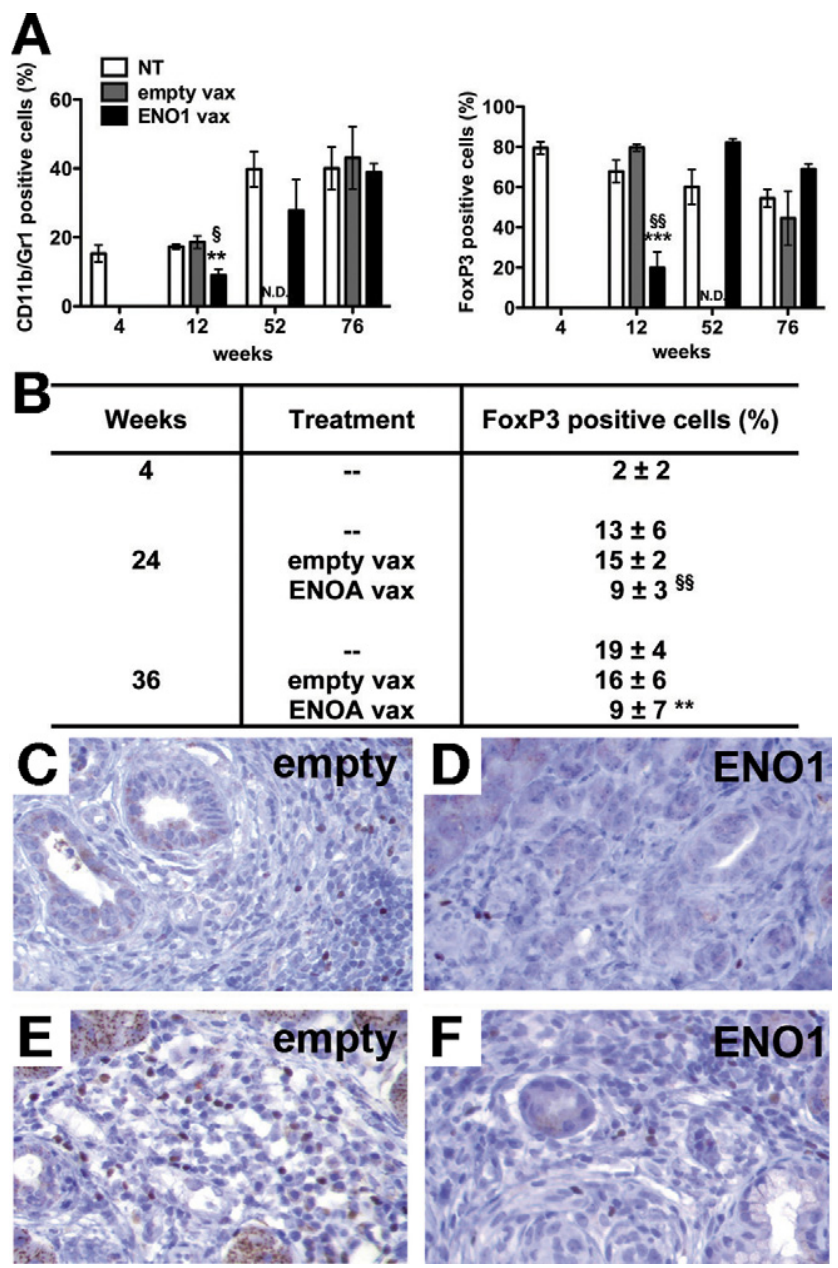

Figure 4. ENO1 vaccination decreased MDSC and FoxP3 ${ }^{+}$cells. (A, left panel) Whole blood from untreated (white bars), empty-vaccinated (gray bars), and ENO1-vaccinated (black bars) mice was stained after red blood cell lysis with anti-CD11b and anti-GR1 mAbs and directly acquired with a FACSCalibur. The percentage of double-positive cells from individual mice is reported as mean \pm SEM; $n=4-8$ per group. (Right pane/) The presence of FoxP3 transcriptional factor was assessed in peripheral blood mononuclear cells from untreated (white bars), empty-vaccinated (gray bars), and ENO1-vaccinated (black bars) mice. The percentage of positive cells between total $\mathrm{CD} 4^{+} / \mathrm{CD} 25^{+}$cells evaluated in each single mouse is reported as mean \pm SEM; $n=4-8$ per group. (B) Quantification of FoxP3 ${ }^{+}$cells in transformed pancreatic ducts $(n=5$ for each group). (C-F) Pancreases from empty- and ENO1-vaccinated mice killed at $(C$ and $D) 24$ and $(E$ and $F) 36$ weeks of age, respectively, were stained with the anti-FoxP3 antibody. ${ }^{\star \star} P<.01$, ${ }^{* \star *} P<.0001$ values significantly different from untreated mice and $\$ p<.01$, $\$ \S P<$ .001 values significantly different from empty-vaccinated mice. 

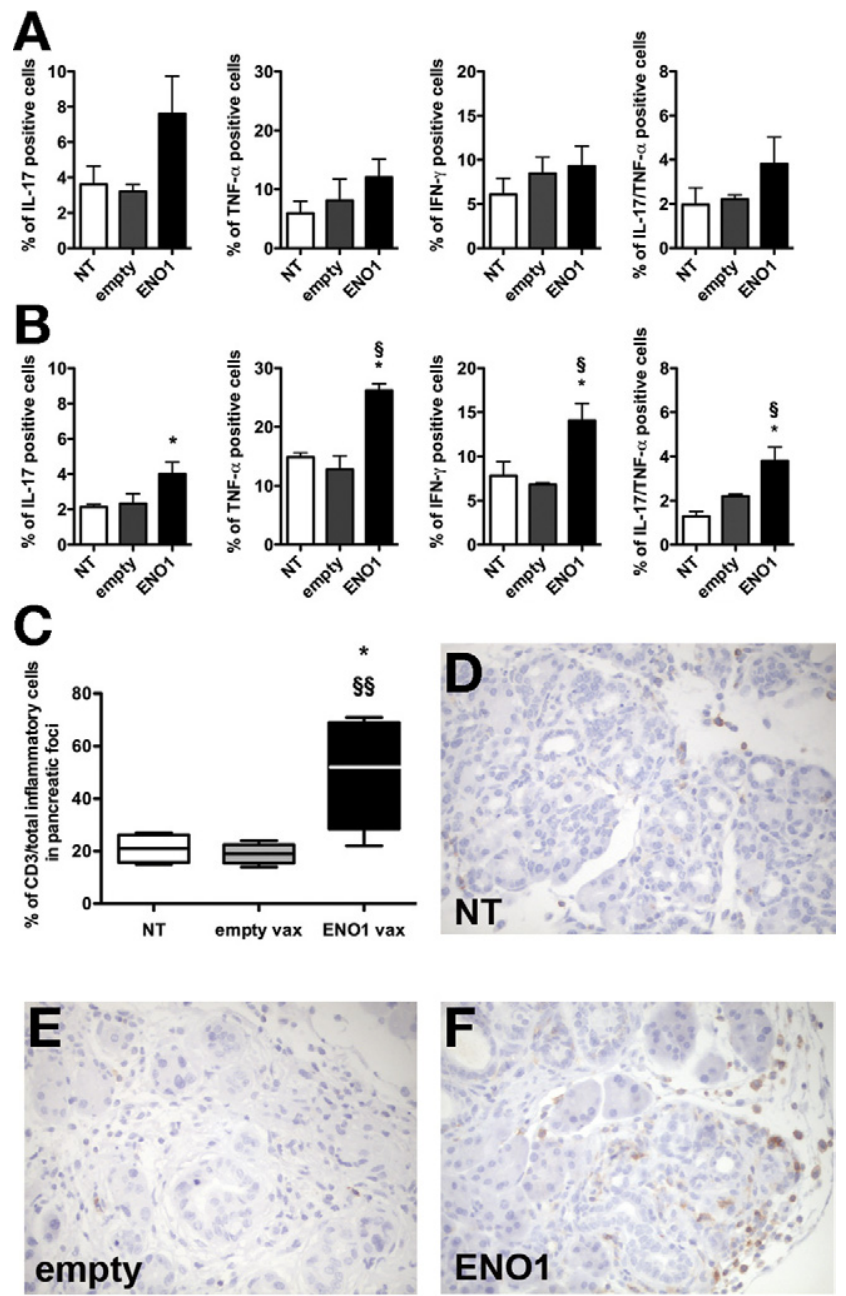

Figure 5. ENO1 vaccination increased IL-17 production. Spleen cells from untreated (white bars), empty-vaccinated (gray bars), and ENO1vaccinated (black bars) mice were stimulated with PMA and ionomycin overnight in the presence of brefeldin $A(A)$ ex vivo and $(B)$ after in vitro stimulation with rENO1 for 7 days and then stained for intracellular cytokines. The percentage of positive cells in total $\mathrm{CD} 4^{+}$cells evaluated in each single mouse is represented as mean \pm SEM; $n=6$ per group. ${ }^{*} P<.05$ values significantly different from untreated mice and $\$ P<.05$ values significantly different from empty-vaccinated mice. (C) Quantification of $\mathrm{CD}^{+}$cells in transformed pancreatic ducts $(n=6-8$ for each group). ( $D-F)$ Pancreases from $(D)$ untreated, $(E)$ empty-vaccinated, and (F) ENO1-vaccinated mice killed at 24 weeks of age, respectively, were stained with the anti-CD3 antibody. ${ }^{*} P<.05$ values significantly different from untreated mice; $\$ \S P<.01$ values significantly different from emptyvaccinated mice.

never for as long as in our study. We show that ENO1 DNA vaccination significantly prolongs survival from 336 to 474 days of age, the longest overall survival ever reported.

In this study, we showed that ENO1-vaccinated mice displayed a higher amount of serum anti-ENO1 IgG and, notably, that they were able to bind the cell surface of murine PDA cells and induce their killing by complementdependent cytotoxicity, which has been proposed as an effector mechanism of antitumor immunity. ${ }^{17,18}$ Induction of anti-ENO1 antibody correlates with the increase of ENO1-specific Th1 and Th17 T cells. The latter, in par- ticular, may be crucially important in helping B cells to produce a pronounced amount of antibodies with preferential isotype class switching to IgG1, IgG2a, IgG2b, and IgG3 ${ }^{19}$ and IFN- $\gamma$ to IgG2a. ${ }^{20}$ Accordingly, we have documented a strong increase in $\mathrm{T}$ lymphocytes that infiltrate the tumor area in ENO1-vaccinated mice. We are currently investigating other anti-tumor mechanisms dependent on anti-ENO1 IgG, especially after the important demonstration by Guo et al that, in addition to surface molecules, proteins hidden within cells can also be attacked by antibodies. ${ }^{21}$ Another possible role is the inhibition of migration of pancreatic tumor cells or MDSCs into the tumor through the ENO1-plasminogen pathway blockade.22,23

An additional important effect of ENO1 vaccination is the significant decrease of MDSCs and Treg cells. The massive secretion of IL-6 in the pancreas of ENO1-vaccinated mice (data not shown) may explain the increase of Th17 rather than FoxP3 ${ }^{+}$cells. ${ }^{24}$ Th17 cells recruit neutrophils and eosinophils, ${ }^{25}$ also present in pancreatic tumor lesions from empty-vaccinated mice (data not shown), and this native immune response partly impeded tumor progression, particularly at the beginning; indeed, at 24 weeks of age, both empty- and ENO1-vaccinated mice presented a lower percentage of transformed pancreatic ducts compared with untreated mice. However, only the combination of the innate and acquired immune responses induced by ENO1 vaccination was able to significantly delay tumor progression. At 36 weeks of age, only ENO1-vaccinated mice showed the lower percentage of transformed pancreatic ducts, and 2 of these 5 mice displayed an almost entirely histologically normal pancreas. Nevertheless, when suppressive immune cells restored percentages similar to those of the controls, progression was no longer counteracted and death ensued. However, it is possible that repeated boosters are required, as for other antigens, to maintain the minimal antigen concentration necessary for adequate effector activation. Thus, all studies aimed to limit suppressor cells by a combination of different strategies are highly applicable, and it is likely that a combined vaccination schedule or different settings might be more efficient. In a genetically engineered mice model of lung adenocarcinoma, vaccination clearly stimulated specific $\mathrm{T}$ cells that soon disappeared; tumor growth, in parallel, was slower at the beginning but then became similar to that in control mice. ${ }^{26}$ This also endorses the great potential of the vaccination even if researchers still have to work on the most effective combination and timing. The choice of xenogenic rather than syngeneic antigen or other kinds of vectors remains open; many studies have shown that human TAA compared with the mouse orthologue, or viral vectors compared with plasmid vectors used to vaccinate tumor-bearing mice, are more efficient in inducing tumor immunity as well as autoimmunity. Thus, xenogenic TAA or viral components aid the immune system in breaking tolerance or ignorance against a "self"-protein. ${ }^{27-29}$ In our case, we have previously shown that peripheral blood mononuclear cells from patients with PDA specifically secreted 
A

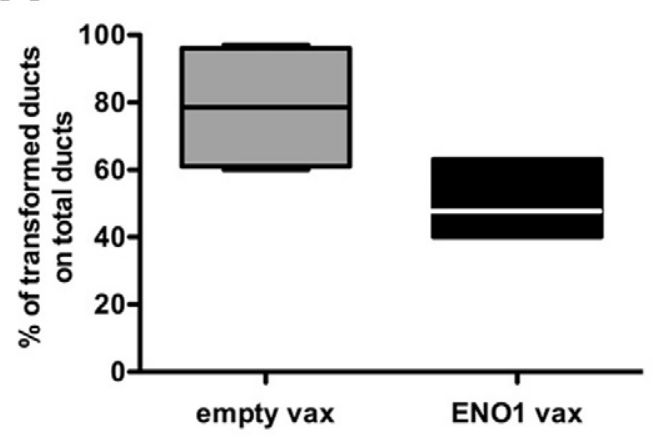

B

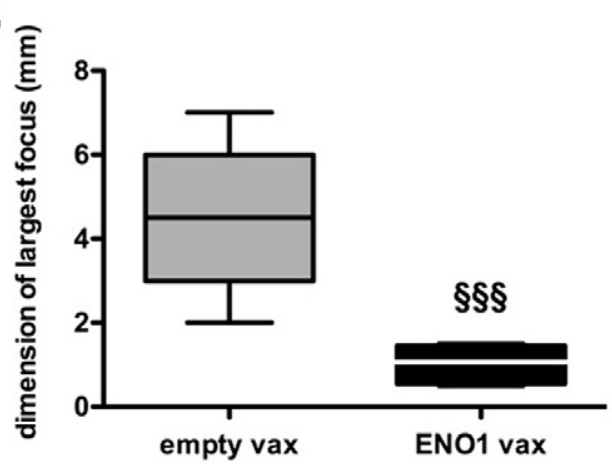

Figure 6. Late ENO1 vaccination significantly delayed progression of PDA. KC mice were vaccinated starting at 32 weeks of age, when PDA was well established, every 3 weeks for a total of 3 rounds. (A) Percentage of transformed ducts in KC mice vaccinated with empty or ENO1 DNA ( $n=7-8$ for each group). (B) Quantification of the largest focus $(\mathrm{mm})$ in the pancreas of mice vaccinated with empty or ENO1 DNA ( $\mathrm{n}=7-8$ for each group). $\$ \$ \$ P .0001$ values compared with empty-vaccinated mice.

IFN- $\gamma$ in response to the syngeneic recombinant protein, and here we show that mice vaccinated with the xenogenic protein produce antibodies against the syngeneic native protein.

Our results are, however, very promising. Few of the many new strategies seem to be effective in prolonging survival beyond 1 year, ${ }^{15}$ while the optimal adjuvant approach after resection is still unclear. ${ }^{30}$ Together these findings suggest that ENO1 vaccination in patients with resected PDA might increase the Th17 population and limit the expansion of MDSCs, leading to an effective immune response that tackles recurrence. In addition, this observation endorses previous data on the effector role of Th17 cells in tumors, ${ }^{31-33}$ even if their specific contribution in PDA remains to be clarified. DNA vaccines could be used in pancreatic cancer as adjuvants to conventional treatments, in the management of minimal residual disease, and as a way of increasing the overall survival of the $80 \%$ of patients with resected PDA who develop recurrences.

Increasing data indicate that chemoimmunotherapy may constitute a new strategy to control tumor progression. ${ }^{34}$ The immune system, indeed, could be elicited in 2 ways by conventional therapies. Some therapeutic programs elicit specific cellular responses that render tumor cell death immunogenic. ${ }^{35}$ Other drugs may have side effects that stimulate the immune system through different mechanisms. Moreover, vaccination against cancerspecific antigens may sensitize a tumor to subsequent chemotherapy, ${ }^{34}$ and in this contest the ENO1 vaccine can also be applied to patients with chemoresistant PDA. Lastly, the chemotherapeutic drug gemcitabine ${ }^{36,37}$ (but not doxorubicin/cyclophosphamide ${ }^{38}$ ) eliminates MD$\mathrm{SCs}^{39}$ and cyclophosphamide eliminates Tregs, ${ }^{40}$ which constitute one of the main immunosuppressive factors in cancer as well as tumor-associated stromal cells, and several strategies targeting them are currently being explored. ${ }^{39,41}$ Very promising, indeed, is the therapeutic efficacy of the ENO1 vaccine observed when the administration protocol is started at 32 to 36 weeks of age. The right drug combination might transform our trend in significant results. Overall, the present data indicate that it may be possible to design adjuvant therapies to elicit anti-ENO1 responses in patients with resected PDA to prevent recurrence or to prolong survival of untreatable patients.

\section{References}

1. Jemal A, Siegel R, Ward E, et al. Cancer statistics, 2008. CA Cancer J Clin 2008;58:71-96.

2. Omura N, Goggins M. Epigenetics and epigenetic alterations in pancreatic cancer. Int J Clin Exp Pathol 2009;2:310-326.

3. Tomaino B, Cappello P, Capello M, et al. Autoantibody signature in human ductal pancreatic adenocarcinoma. J Proteome Res 2007; 6:4025-4031.

4. Tomaino B, Cappello P, Capello M, et al. Circulating autoantibodies to phosphorylated alpha-enolase are a hallmark of pancreatic cancer. J Proteome Res 2011;10:105-112.

5. Cappello $P$, Tomaino $B$, Chiarle R, et al. An integrated humoral and cellular response is elicited in pancreatic cancer by alpha-enolase, a novel pancreatic ductal adenocarcinoma-associated antigen. Int J Cancer 2009;125:639-648.

6. Capello M, Ferri-Borgogno S, Cappello P, et al. $\alpha$-enolase: a promising therapeutic and diagnostic tumor target. FEBS J 2011;278: 1064-1074.

7. Pancholi V. Multifunctional alpha-enolase: its role in diseases. Cell Mol Life Sci 2001;58:902-920.

8. Hingorani SR, Petricoin EF, Maitra A, et al. Preinvasive and invasive ductal pancreatic cancer and its early detection in the mouse. Cancer Cell 2003;4:437-450.

9. Hruban RH, Adsay NV, Albores-Saavedra J, et al. Pathology of genetically engineered mouse models of pancreatic exocrine cancer: consensus report and recommendations. Cancer Res 2006; 66:95-106.

10. Giallongo A, Feo S, Moore R, et al. Molecular cloning and nucleotide sequence of a full-length cDNA for human alpha enolase. Proc Natl Acad Sci U S A 1986;83:6741-6745.

11. Giovarelli M, Cappello P, Forni G, et al. Tumor rejection and immune memory elicited by locally released LEC chemokine are associated with an impressive recruitment of APCs, lymphocytes, and granulocytes. J Immunol 2000;164:3200-3206.

12. Clark CE, Hingorani SR, Mick R, et al. Dynamics of the immune reaction to pancreatic cancer from inception to invasion. Cancer Res 2007;67:9518-9527. 
13. Martinez GJ, Nurieva RI, Yang $X O$, et al. Regulation and function of proinflammatory TH17 cells. Ann N Y Acad Sci 2008;1143:188211.

14. Kryczek I, Banerjee M, Cheng $\mathrm{P}$, et al. Phenotype, distribution, generation, and functional and clinical relevance of Th17 cells in the human tumor environments. Blood 2009;114:1141-1149.

15. Laheru D, Jaffee EM. Immunotherapy for pancreatic cancerscience driving clinical progress. Nat Rev Cancer 2005;5:459 467.

16. Hingorani SR, Wang L, Multani AS, et al. Trp53R172H and KrasG12D cooperate to promote chromosomal instability and widely metastatic pancreatic ductal adenocarcinoma in mice. Cancer Cell 2005;7:469-483.

17. Weiner LM, Murray JC, Shuptrine CW. Antibody-based immunotherapy of cancer. Cell 2012;148:1081-1084.

18. Shuptrine CW, Surana R, Weiner LM. Monoclonal antibodies for the treatment of cancer. Semin Cancer Biol 2012;22:3-13.

19. Mitsdoerffer $M$, Lee $Y$, Jager $A$, et al. Proinflammatory $T$ helper type 17 cells are effective B-cell helpers. Proc Natl Acad Sci U S A 2010;107:14292-14297.

20. Hasbold J, Hong JS, Kehry MR, et al. Integrating signals from IFN-gamma and IL-4 by B cells: positive and negative effects on CD40 ligand-induced proliferation, survival, and division-linked isotype switching to IgG1, IgE, and IgG2a. J Immunol 1999;163: 4175-4181.

21. Guo K, Li J, Tang JP, et al. Targeting intracellular oncoproteins with antibody therapy or vaccination. Sci Transl Med 2011;3:99ra85.

22. Lopez-Alemany R, Longstaff C, Hawley S, et al. Inhibition of cell surface mediated plasminogen activation by a monoclonal antibody against alpha-enolase. Am J Hematol 2003;72:234-242.

23. Wygrecka M, Marsh LM, Morty RE, et al. Enolase-1 promotes plasminogen-mediated recruitment of monocytes to the acutely inflamed lung. Blood 2009;113:5588-5598.

24. Gnerlich JL, Mitchem JB, Weir JS, et al. Induction of Th17 cells in the tumor microenvironment improves survival in a murine model of pancreatic cancer. J Immunol 2010;185:4063-4071.

25. Fujita K, Ewing CM, Sokoll $\amalg$, et al. Cytokine profiling of prostatic fluid from cancerous prostate glands identifies cytokines associated with extent of tumor and inflammation. Prostate 2008;68: 872-882.

26. DuPage M, Cheung AF, Mazumdar C, et al. Endogenous T cell responses to antigens expressed in lung adenocarcinomas delay malignant tumor progression. Cancer Cell 2011;19:72-85.

27. Weber LW, Bowne WB, Wolchok JD, et al. Tumor immunity and autoimmunity induced by immunization with homologous DNA. J Clin Invest 1998;102:1258-1264.

28. Bowne WB, Srinivasan R, Wolchok JD, et al. Coupling and uncoupling of tumor immunity and autoimmunity. J Exp Med 1999;190: 1717-1722.

29. Overwijk WW, Tsung A, Irvine KR, et al. gp100/pmel 17 is a murine tumor rejection antigen: induction of "self"-reactive, tumoricidal T cells using high-affinity, altered peptide ligand. J Exp Med 1998;188:277-286.

30. Li J, Merl MY, Chabot J, et al. Updates of adjuvant therapy in pancreatic cancer: where are we and where are we going? Highlights from the "2010 ASCO Annual Meeting." Chicago, IL, USA. June 4-8, 2010. JOP 2010;11:310-312.

31. Kryczek I, Wei S, Szeliga W, et al. Endogenous IL-17 contributes to reduced tumor growth and metastasis. Blood 2009;114:357359.

32. Martin-Orozco N, Muranski $\mathrm{P}$, Chung $\mathrm{Y}$, et al. $\mathrm{T}$ helper 17 cells promote cytotoxic $\mathrm{T}$ cell activation in tumor immunity. Immunity 2009;31:787-798.
33. Zou W, Restifo NP. T(H)17 cells in tumour immunity and immunotherapy. Nat Rev Immunol 2010;10:248-256.

34. Zitvogel L, Apetoh L, Ghiringhelli F, et al. Immunological aspects of cancer chemotherapy. Nat Rev Immunol 2008;8:59-73.

35. Kroemer G, Zitvogel L. Death, danger, and immunity: an infernal trio. Immunol Rev 2007;220:5-7.

36. Suzuki E, Kapoor V, Jassar AS, et al. Gemcitabine selectively eliminates splenic Gr-1+/CD11b + myeloid suppressor cells in tumor-bearing animals and enhances antitumor immune activity. Clin Cancer Res 2005;11:6713-6721.

37. Ko HJ, Kim YJ, Kim YS, et al. A combination of chemoimmunotherapies can efficiently break self-tolerance and induce antitumor immunity in a tolerogenic murine tumor model. Cancer Res 2007 67:7477-7486.

38. Diaz-Montero CM, Salem ML, Nishimura MI, et al. Increased circulating myeloid-derived suppressor cells correlate with clinical cancer stage, metastatic tumor burden, and doxorubicin-cyclophosphamide chemotherapy. Cancer Immunol Immunother 2009; 58:49-59.

39. Gabrilovich DI, Nagaraj S. Myeloid-derived suppressor cells as regulators of the immune system. Nat Rev Immunol 2009;9:162174.

40. Sistigu A, Viaud S, Chaput N, et al. Immunomodulatory effects of cyclophosphamide and implementations for vaccine design. Semin Immunopathol 2011;33:369-383.

41. Kraman M, Bambrough PJ, Arnold JN, et al. Suppression of antitumor immunity by stromal cells expressing fibroblast activation protein-alpha. Science 2010;330:827-830.

Received April 12, 2012. Accepted January 10, 2013.

\section{Reprint requests}

Address requests for reprints to: Francesco Novelli, PhD, Center for Experimental Research and Medical Studies, University of Turin, Via Cherasco 15, 10125 Turin, Italy. e-mail: franco.novelli@unito.it; fax: (39) 0116336887 .

\section{Acknowledgments}

The authors thank Dr D. Tuveson and Dr A. Amedei for scientific discussion, Dr John Iliffe and Radhika Srinivasan for critically reading the manuscript, and M.-S. Scalzo, G. Barutello, and R. Curto for technical help. In addition, authors thank to FIRMS for helping in funding's management.

\section{Conflicts of interest}

The authors disclose no conflicts.

\section{Funding}

Supported by the Associazione Italiana per la Ricerca sul Cancro to RC and FN (AIRC IG n.11643, $5 \times 1000$ n.12182); Ministero dell'Istruzione, dell'Università e della Ricerca (MIUR), Progetti di Rilevante Interesse Nazionale (PRIN); Ministero della Sanità: Progetto Integrato Oncologia; Regione Piemonte: Ricerca Industriale e Sviluppo Precompetitivo (ONCOPROT), Ricerca Industriale "Converging Technologies" (BIOTHER), Progetti strategici su tematiche di interesse regionale o sovra regionale (IMMONC), Ricerca Sanitaria Finalizzata, Ricerca Sanitaria Applicata; and European Community, European Community's Seventh Framework Programme (FP7/2007-2013 grant agreement no. 256974 to FN) and FP7 ERC2009- StG (Proposal No. 242965 "Lunely" to RC) and the International Association for Cancer Research (AICR) to RC. P.C. was supported by a fellowship from Fondazione Italiana Ricerca sul Cancro (FIRC). 